\title{
Towards Using the Spatio-temporal Properties of Eye Movements to Classify Visual Field Defects
}

\author{
Alessandro Grillini \\ Department of Ophthalmology, UMCG \\ Groningen, The Netherlands \\ a.grillini@rug.nl \\ Rijul S. Soans \\ Department of Electrical Engineering, IIT Delhi \\ New Delhi, India \\ rijul.soans@ee.iitd.ac.in
}

\author{
Daniel Ombelet \\ Faculty of Medical Sciences, University of Groningen \\ Groningen, The Netherlands \\ d.ombelet@student.rug.nl \\ Frans W. Cornelissen \\ Department of Ophthalmology, UMCG \\ Groningen, The Netherlands \\ f.w.cornelissen@ucmg.nl
}

\begin{abstract}
Perimetry-assessment of visual field defects (VFD)-requires patients to be able to maintain a prolonged stable fixation, as well as to provide feedback through motor response. These aspects limit the testable population and often lead to inaccurate results. We hypothesized that different VFD would alter the eye-movements in systematic ways, thus making it possible to infer the presence of VFD by quantifying the spatio-temporal properties of eye movements. We developed a tracking test to record participant's eyemovements while we simulated different gaze-contingent VFD. We tested 50 visually healthy participants and simulated three common scotomas: peripheral loss, central loss and hemifield loss. We quantified spatio-temporal features using cross-correlogram analysis, then applied cross-validation to train a decision tree algorithm to classify the conditions. Our test is faster and more comfortable than standard perimetry and can achieve a classifying accuracy of $\sim 90 \%$ (True Positive Rate $=\sim 98 \%$ ) with data acquired in less than 2 minutes.
\end{abstract}

\section{CCS CONCEPTS}

-Applied computing $\rightarrow$ Consumer health; • Computing methodologies $\rightarrow$ Classification and regression trees;

\section{KEYWORDS}

gaze-contingency, simulations, features classification, tracking, visual field, cross-correlation, screening

\section{ACM Reference Format:}

Alessandro Grillini, Daniel Ombelet, Rijul S. Soans, and Frans W. Cornelissen. 2018. Towards Using the Spatio-temporal Properties of Eye Movements to Classify Visual Field Defects. In ETRA '18: 2018 Symposium on Eye Tracking Research and Applications, fune 14-17, 2018, Warsaw, Poland. ACM, New York, NY, USA, 5 pages. https://doi.org/10.1145/3204493.3204590

Permission to make digital or hard copies of part or all of this work for personal or classroom use is granted without fee provided that copies are not made or distributed for profit or commercial advantage and that copies bear this notice and the full citation on the first page. Copyrights for third-party components of this work must be honored.

For all other uses, contact the owner/author(s).

ETRA '18, June 14-17, 2018, Warsaw, Poland

(c) 2018 Copyright held by the owner/author(s).

ACM ISBN 978-1-4503-5706-7/18/06.

https://doi.org/10.1145/3204493.3204590

\section{INTRODUCTION}

Detecting the presence of visual field defects (VFD) at an early stage is a critical aspect of diagnosing many ophthalmic and neurological disorders (i.e. glaucoma, macular degeneration, post-stroke visual loss). There are several ways to assess the presence of VFD. Two of the most commonly used methods are Standard Automated Perimetry (SAP) and Frequency Doubling Technology (FDT) perimetry. However, there are a number of challenges when it comes to visual field testing. Firstly, most test versions of SAP require concentration for a prolonged period of time ( 10 $\mathrm{min})$-this can render the task tedious for some participants and impossible for others. FDT instead takes approx. 2 min but is relatively insensitive to minor defects. Secondly, in both SAP and FDT, the participant is required to maintain a stable fixation. Failing to do so results in fixation loss and-consequently-an extended measurement of time or less reliable results. Thirdly, all current perimetric techniques require the patients to provide their feedback through motor response. While being no more than nuisances to many participants, these limitations prevent the clinical assessment of some groups of patients. In particular, elderly people and children are affected by these limitations. Modern perimetry has its roots in psychophysical methodology. The participant has to detect a stimulus and answer the simple question: "Did you see it?" However, the continuous need to question the participant repeatedly is one of the reasons that extend the assessment period. A solution could be continuously interrogating the visual system's ability to detect the presence of the stimulus. If participants are able to detect a stimulus, they also have information about its approximate position. While if the stimulus is less visible or invisible, its positional information will also deteriorate or be absent. This suggests that an alternative means of assessing the visual system lies in the continuous tracking of a stimulus' position. Indeed, [Bonnen et al. 2015] showed that the spatial sensitivity measured by continuous tracking (performed with a computer mouse) correlates well with to the one measured by means of traditional psychophysics. However, the human visual system has a "built-in tracking device"-i.e. the eyes-of which the performance is very directly linked to the quality of visual processing. For this reason, we studied the possibility of using eye-movement properties assessed by means of a continuous tracking task to screen for the presence of VFD. We hypothesize that different VFD will alter the spatio-temporal eye movements in a specific and measurable 
way. We expect that the presence of a VFD will affect one's ability to track a stimulus. First, if the sensitivity of the central visual field or the entire visual system is reduced, we can expect prolonged processing time and therefore delayed tracking performance. Second, if the tracking stimulus completely disappears because of a scotoma, it will take time to find it again and pursue tracking, delaying the performance and increasing the spatial error. Given these predicted alterations, we expect that it should also be possible to identify the underlying defect based on the spatio-temporal characteristics of the eye movements by training a machine-learning classifier.

\section{METHODS}

\subsection{Participants and Ethical Clearance}

We tested 50 healthy participants and one glaucoma patient with peripheral visual field loss, previously assessed with SAP. All had normal or corrected-to-normal visual acuity, verified prior to data collection with "FrACT" [Bach 2007]. The study followed the tenets of the Declaration of Helsinki. The ethics board of the Psychology Department of the University of Groningen approved the study protocol. All participants provided written informed consent before participation.

\subsection{Materials and Procedures}

Participants performed a visual tracking task while being subjected to different simulations of VFD. No simulation was applied to the glaucoma patient. The conditions were sorted with stratified randomization. The gaze position was constantly monitored and recorded.

2.2.1 Apparatus. The experiment was designed and conducted with custom-made scripts in MATLAB using Psychtoolbox (Pelli 1997) and Eyelink Toolbox [Cornelissen et al. 2002]. The data was acquired with an eye-tracker Eyelink 1000 (SR-Research, Kanata, Ontario, Canada) with a sampling frequency of $1 \mathrm{KHz}$, downsampled to $240 \mathrm{~Hz}$ to match the refresh rate of the stimulus display monitor Zowie xl2540 (BenQ, Taipei, Taiwan).

2.2.2 Tracking Stimulus. The stimulus comprises of a Gaussian luminance blob (FWHM $=0.5$ degrees of visual angle) moving in a $2 \mathrm{D}$ random-walk path. In each trial, the stimulus could be presented either at a high (50\%) or low (5\%) contrast. The random-walk path could be smooth (continuous) or saccadic (random displacement applied every $2 \mathrm{sec}$ ). The instantaneous speeds for every frame of the stimulus presentation were randomly drawn from a standard normal distribution, then multiplied by two different gains for the horizontal and vertical components (drawn independently). Both the resulting distributions are zero-mean and have standard deviations of $\pm 64.45 \mathrm{deg} / \mathrm{sec}$ (horizontal) and $\pm 32.23 \mathrm{deg} / \mathrm{sec}$ (vertical).

2.2.3 Gaze-contingent simulated VFD. The simulations were obtained by superimposing in real-time $(240 \mathrm{~Hz}, 4 \mathrm{~ms}$ delay) a uniform grey area to the current position of the participant's gaze. The shape and size of the simulated VFD were modelled after the typical scotoma resulting from three common ophthalmologic disorders: age-related macular degeneration (central loss), late stage glaucoma (peripheral loss) and hemianopia (hemifield loss) (Figure 1).
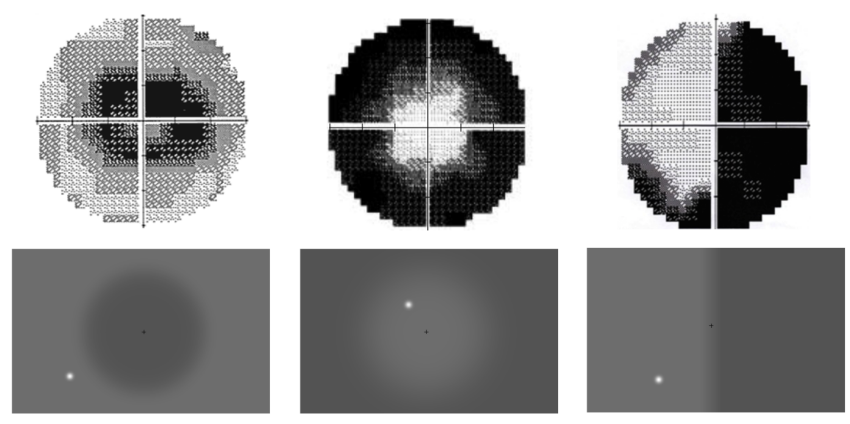

Figure 1: Top row: examples of scotoma represented by visual field maps. From left to right: central loss, peripheral loss, hemifield loss. Bottom row: simulated gaze-contingent VFD (darker for visualization purposes only).

For each combination of conditions (pursuit $\times$ contrast $\times$ VFD), we performed 6 tracking tasks of $20 \mathrm{sec}$ each.

\subsection{Spatio-temporal Features Extraction}

The data was analyzed according to the Eye-Movement Correlogram technique described by [Mulligan et al. 2013]: the instantaneous velocities of stimulus and eye are thresholded to separate the saccadic component $(v(t)>30 \mathrm{deg} / \mathrm{sec})$ from the smooth pursuit component, then cross-correlated independently and averaged across trials. Finally, we computed the instantaneous positional error as the Euclidean Distance between the eye and stimulus positions.

\section{Abbreviations:}

CCG1 Cross-correlogram between the smooth velocity of the eye and stimulus;

CCG2 Cross-correlogram between saccadic velocity and positional error;

PDD Probability density distribution of positional error.

The 10 spatio-temporal features that we used to train the classifier are: Amplitude, $\mu, \sigma$ and $\mathrm{R}^{2}$ of the Gaussian fits to CCG1 and to PDD respectively, peak and delay of the CCG2. All features were determined separately for the horizontal and the vertical components of the eye-movements. Therefore, we obtained a dataset comprising 80 features ( 10 features $\times 2$ axis $\times 2$ contrast levels $\times 2$ pursuit modalities) per 200 entries (50 participants $\times 4$ conditions).

\subsection{Features Classification}

We used Decision Trees (DTs) [Breiman et al. 1984] for discriminating between the various VFD and controls. Since DTs have an inherent feature selection capability [Weiss and Indurkhya 1998], we used them also for reducing the dimensionality of our dataset. DTs split a set of labelled data at each node into smaller branches before eventually converging onto one of the possible decisions. In our context, the initial node is an 80-dimensional feature space that is constituted from the 80 features obtained from the crosscorrelogram analysis. The feature space is then split into smaller subspaces at every subsequent node based on an "impurity criterion". The decision criterion that we use is the "Gini's Diversity 

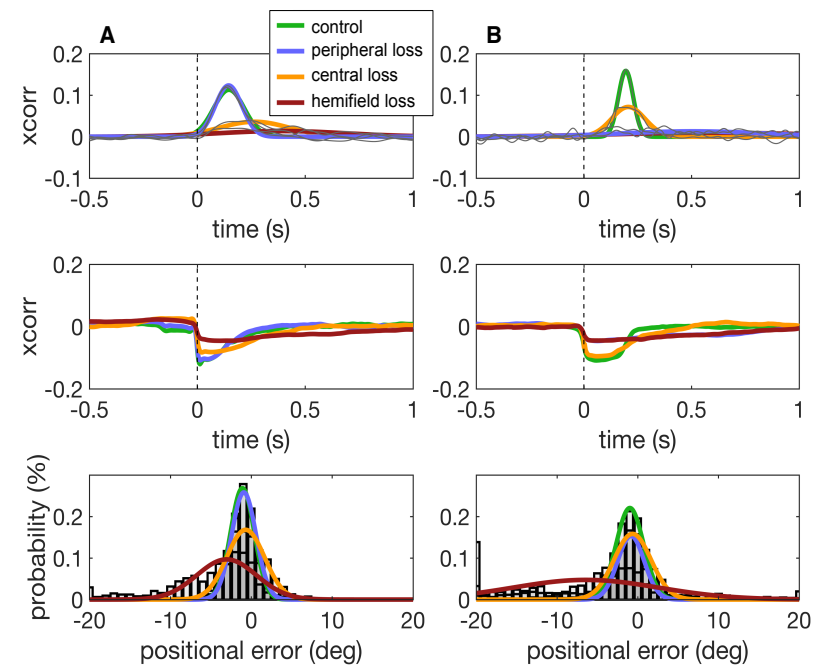

Figure 2: Example of single participant results. The spatiotemporal features are extracted from the resulting CCGs and PDD.

Index" (GDI) given as follows:

$$
1-\sum f^{2}(i)
$$

Where, $f(i)$ is the fraction of the number of samples in the training set for class $i$ that reaches a particular node ( $i$ being an index for control, peripheral loss, central loss and hemifield loss). If a node has samples of only 1 class coming into it, then the GDI has a value of 0 and the node is termed as a pure node. Consequently, that node is assigned as a class, thereby completing the decision. On the other hand, if a node has a mixture of samples coming from various classes, then the GDI has some positive value and the node is split in such a way so as to minimize the GDI after the split. Finally, to estimate the classifier's performance, we used a 10-fold cross-validation scheme wherein the entire dataset is randomly partitioned into 10 subsets of equal size. Subsequently, 9 subsets constitute the training set, and the remaining 1 set is used as test set. This process is then repeated until every subset has been used once as a test set. The estimated total accuracy is the average of the accuracies measured after each repetition.

\section{RESULTS}

\subsection{Cross-Correlograms and Probability Density Distributions}

Figure 2 shows an example of analyzed data for one participant in the smooth pursuit (panel A) and saccadic pursuit (panel B) tracking modalities, in the control and the three VFD simulation conditions, respectively. The top row shows CCG1 (stimulus $\times$ eye velocities). The Gaussian fits for the simulated VFD (in blue, yellow and red) are both delayed and shallower compared to those for the control condition (in green), indicating reduced performance in both pursuit modalities (A and B).

The mid-row shows CCG2 (positional error $\times$ saccadic velocity). The peak of the correlation at time point 0 is negative because the

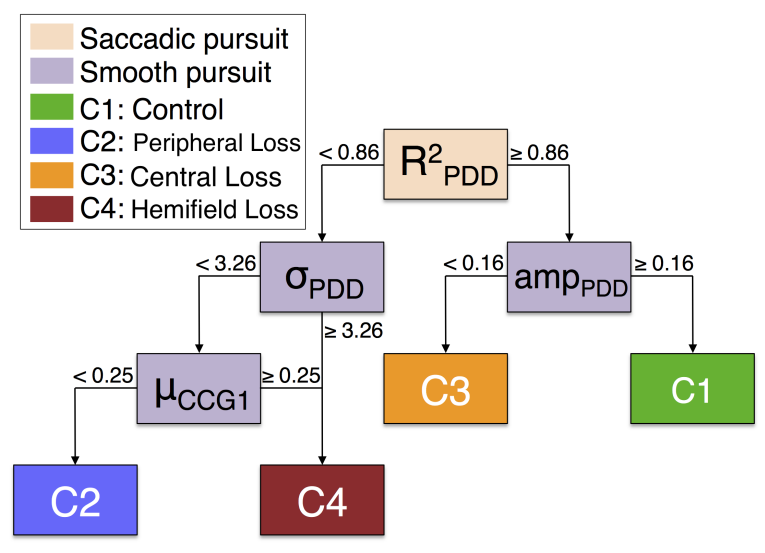

Figure 3: The Decision Tree (DT) for classifying VFD based on spatio-temporal characteristics of eye-movements. The features used in the classification are $R^{2}$, standard deviation and amplitude of PDD and the mean of CCG1. The DT finds the threshold values in these features that achieve the best separation between classes.

saccadic velocity is always of the opposite sign of the positional error: if the eye is overshooting to the right (positive positional error) correction requires making a saccade to the left and vice-versa. Also, in this case, the CCG gets shallower in the VFD conditions, indicating longer correction time. The bottom row shows the positional error (PDD). Again, the VFD lead to shallower Gaussian fits indicating performance deterioration. Noticeably, in the case of hemifield loss, we also observe a large shift of the mean error in the direction of the scotoma.

\subsection{Features Selection and Classifier Performance}

Figure 3 shows the DT resulting from the highest accuracy achieved (91.5\%). The most noticeable thing is the large reduction in dimensionality: from the initial 80 features only 4 "survived". Moreover, we observed that amongst the different features, the vertical axis and the high contrast ones were always absent, thus not contributing to the classification. Therefore, these feature levels are omitted from the graphical representation shown in Figure 3.

Figure 4-A shows the accuracy of the classifier as a function of the duration of the data collection. Even just 20 seconds of data collection is sufficient to yield an accuracy level above $75 \%$. The accuracy stabilizes at around $90 \%$ for durations above $1 \mathrm{~min}$. Finally, Figure 4-B shows the True Positive Rate and False Negative Rate (TPR and FNR) (i.e. "when a participant really has a disorder, how often our test classifies it correctly?"), measures that are of paramount importance in clinical screening tests. We found a very high TPR for controls (98\%), and relatively lower TPRs for each VFD conditions. 

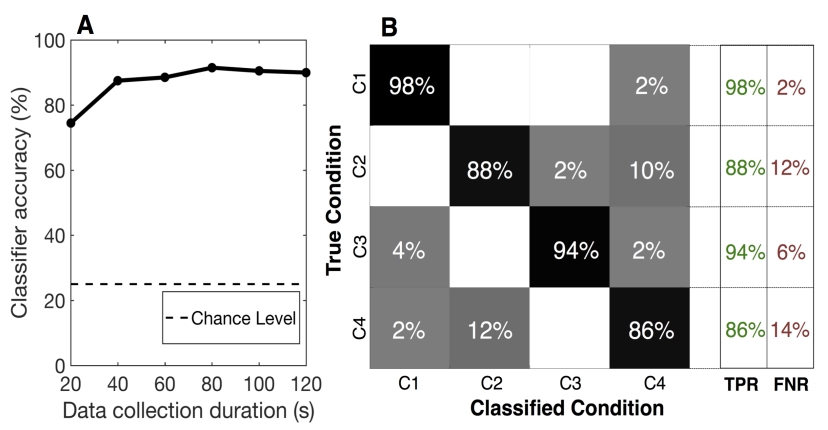

Figure 4: A. Classifier accuracy. B. True Positive Rate and False Negative Rate. C1: control; C2: central loss; C3: peripheral loss; C4: hemifield loss.

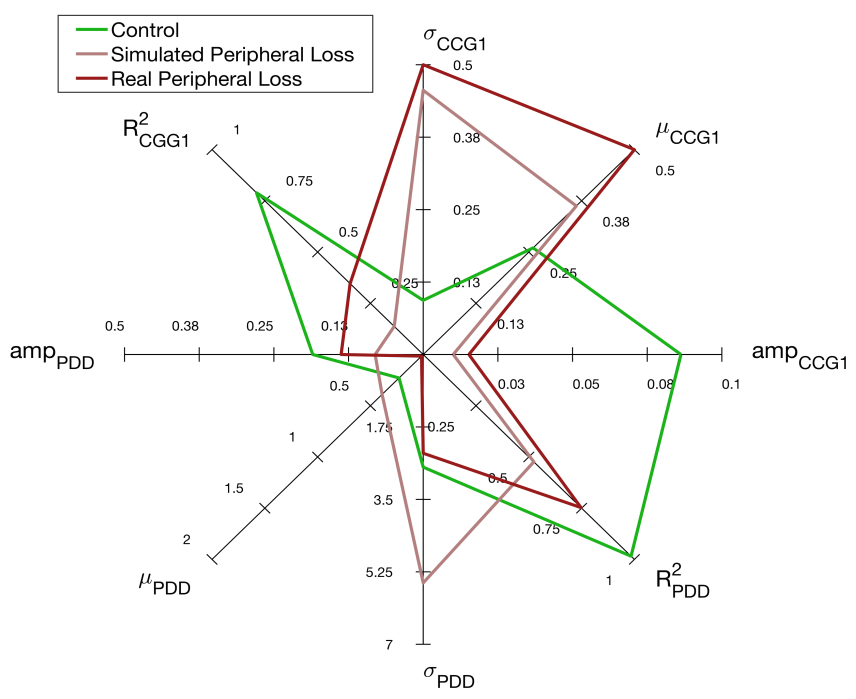

Figure 5: Patterns obtained from spatio-temporal features. The patient pattern (in red) resembles the average pattern obtained from simulated VFD, while the control group is clearly separated.

\subsection{Comparison between simulated and real peripheral loss}

Figure 5 shows the comparison between control group, simulated peripheral loss group and a single patient previously diagnosed with peripheral visual field loss due to glaucoma. Only parameters derived from the saccadic pursuit are shown since for peripheral loss the smooth pursuit condition results are very similar to controls (see Figure 2, top row, the overlap between blue and green lines).

The pattern obtained with the glaucoma patient closely resembles the simulated peripheral loss group pattern, rather than the control group one.

\section{DISCUSSION}

Our main finding is that the spatio-temporal properties of eye movements change when a simulated VFD interferes with the tracking task. Further, these changes appear to be systematic and specific to each VFD. Moreover, we show that a classification approach based on an analysis of spatio-temporal properties of eye movements is feasible.

\subsection{Relevant and irrelevant screening conditions}

Following the CCG and PDD analysis, we can observe that the differences between smooth pursuit and saccadic pursuit are more prominent in the peripheral loss condition and, partially, in the hemifield loss one. The most likely explanation is that during the saccadic task, the stimulus "jumping" into the scotomatous region made this task much more difficult. On the other hand, having their central vision spared, the participants were able to track the stimulus with ease in the smooth pursuit condition. This aspect needs to be taken into account if the goal is to develop a comprehensive screening test, and it is further confirmed by the DT results: both pursuit tasks contributed to a correct classification, while other tested features turned out to be uninformative.

\subsection{Clinical implications and limitations}

A fast screening test that neither requires a manual motor response nor stable fixation potentially holds a major step forward from current SAP techniques. However, the present study was limited in various ways. First, we used simulations that are a simplified version of real VFD. Although our preliminary clinical results are encouraging, a thorough evaluation of patients with actual VFD, and a comparison to other perimetric techniques will be the next necessary step. Moreover, it has previously been shown that with either simulated or real defects, the eye movements will adapt to accommodate for various problems [Coeckelbergh et al. 2002; Schuett et al. 2009; Walsh and Liu 2014]. However, relying entirely on these accommodations in order to detect VFD is still a relatively unexplored field. A previous study managed to detect macular degeneration from the scan-path generated during natural viewing behaviour [Crabb et al. 2014]. Another limitation is the dependence on eye-tracker calibration, a procedure that can be difficult for a patient with severe central vision loss. Although the temporal features are not affected, since they are computed on relative displacements rather than absolute position, the spatial features are particularly sensitive to measurement bias. The overall accuracy achieved by the classifier, tied to the very short amount of time needed, make this potentially appealing to implement this as a clinical test. However, it is important to note that the number of participants was relatively small, compared to standard clinical and normative datasets. Furthermore, each participant served both as control and as "patient", hence removing a part of inter-subject variability typically present in the general population. More data and testing in an actual clinical setting would be required to generalize our results.

\section{CONCLUSIONS}

We have developed a new test for screening for visual field defects that is entirely based on evaluating eye movement behaviour in response to an extremely simple visual task. This way, it improves on some of the most critical aspects of current perimetric techniques: it 
is faster and therefore hardly fatiguing, it is less attentionally taxing, does neither require stable fixation nor manual motor responses. For these reasons, we consider the present proof-of-concept sufficiently promising to verify its ability to serve as a screening tool for visual field defects in clinical practice.

\section{ACKNOWLEDGMENTS}

This project has received funding from the Graduate School of Medical Sciences, Rijksuniversiteit Groningen and the European Union's Horizon 2020 research and innovation programme under the Marie Sklodowska-Curie grant agreement No 641805 to FWC. RSS received a stipend from the Graduate School of Medical Sciences Abel Tasman program to work on the project. We are thankful to Nomdo Jansonius for his advice and help.

\section{REFERENCES}

Michael Bach. 2007. The Freiburg Visual Acuity Test-variability unchanged by post-hoc re-analysis. Graefes Arch. Clin. Exp. Ophthalmol. 245, 7 (July 2007), 965-971.

Kathryn Bonnen, Johannes Burge, Jacob Yates, Jonathan Pillow, and Lawrence K Cormack. 2015. Continuous psychophysics: Target-tracking to measure visual sensitivity. F. Vis. 15, 3 (March 2015).

Leo Breiman, Jerome Friedman, Charles J Stone, and R A Olshen. 1984. Classification and Regression Trees. Chapman and Hall/CRC.

Tanja R M Coeckelbergh, Frans W Cornelissen, Wiebo H Brouwer, and Aart C Kooijman 2002. The effect of visual field defects on eye movements and practical fitness to drive. Vision Res. 42, 5 (2002), 669-677.

Frans W Cornelissen, Enno M Peters, and John Palmer. 2002. The Eyelink Toolbox: eye tracking with MATLAB and the Psychophysics Toolbox. Behav. Res. Methods Instrum. Comput. 34, 4 (Nov. 2002), 613-617.

David P Crabb, Nicholas D Smith, and Haogang Zhu. 2014. What's on TV? Detecting age-related neurodegenerative eye disease using eye movement scanpaths. Front. Aging Neurosci. 6 (2014)

Jeffrey B Mulligan, Scott B Stevenson, and Lawrence K Cormack. 2013. Reflexive and voluntary control of smooth eye movements. In Human Vision and Electronic Imaging XVIII.

Susanne Schuett, Robert W Kentridge, Josef Zihl, and Charles A Heywood. 2009 Adaptation of eye-movements to simulated hemianopia in reading and visual exploration: Transfer or specificity? Neuropsychologia 47, 7 (2009), 1712-1720.

David V Walsh and Lei Liu. 2014. Adaptation to a simulated central scotoma during visual search training. Vision Res. 96 (2014), 75-86.

Sholom M Weiss and Nitin Indurkhya. 1998. Predictive Data Mining: A Practical Guide. Morgan Kaufmann. 